\title{
Suspended Tunnel Junction Bolometers For Terahertz Range
}

\author{
Andrey V. Timofeev ${ }^{*}, 1$, Panu Helistö ${ }^{1}$, Leif Grönberg ${ }^{1}$, Arttu Luukanen $^{1,2}$, Heikki Seppä ${ }^{1}$ and \\ Juha Hassel ${ }^{1}$ \\ ${ }^{I}$ VTT Technical Research Centre of Finland, P.O. Box 1000, FI-02044 VTT, Espoo, Finland \\ ${ }^{2}$ Millilab, VTT, Tietotie 3, FIN-02044 Espoo, Finland
}

\begin{abstract}
Implementation of high resolution passive THz cameras operated at cryogenic temperatures of a few Kelvin benefits from a large number of pixels. At present, building a cryogenic multiplexed read-out circuit represents a challenging task. Here we propose and demonstrate broadband niobium-based tunnel junction bolometers operating in equilibrium regime at $4 \mathrm{~K}$, and read out with a room temperature amplifier with feedback. The implemented scheme allows independent impedance matching for the $\mathrm{THz}$ antenna and for the readout. Electrical feedback in the readout scheme eliminates the need for magnetic fields to suppress the supercurrent of the junction. We present electrical and preliminary optical measurements of the detectors. We also analyze a possible scheme for time-domain multiplexing.
\end{abstract}

Keywords: THz imaging, bolometers, SIS junction.

\section{INTRODUCTION}

Passive real-time $\mathrm{THz}$ imaging based on scanning optics with single detectors or small arrays of hot-spot bolometers have been demonstrated recently [1-3]. Scanning compromises resolution and adds complexity. Therefore "staring" focal plane arrays with static optics are preferable. With increasing number of pixels and limitations set by cryogenics multiplexed readout becomes a necessity. This in turn sets requirements for the detectors. For time-domain multiplexing a bolometer should have a sufficiently low noise equivalent power (NEP) together with sufficient power output to overcome the postamplifier noise. In case of semiconducting readout amplifiers this typically requires relatively large dynamic impedance of the detector element to achieve noise matching. Superconducting tunnel junction (SIS) detectors are well-known $\mathrm{THz}$ radiation detectors anticipated to be suitable for large multiplexable arrays [4-7]. Direct photon assisted tunneling based SIS-detectors offer good sensitivity and noise performance, but within a limited bandwidth $[4,5]$ due to antenna impedance-matching issues. Broadband SIS detectors based on Cooper-pair breaking have been demonstrated recently [6]. They detect both $\mathrm{THz}$ photons and $\mathrm{THz}$ phonons generated by incident radiation and they function efficiently at sub-Kelvin temperatures. For practical $\mathrm{THz}$ scanners simpler cryogenics would be a great benefit [1]. Here we introduce broadband SIS-bolometers operating in an equilibrium mode for a $4 \mathrm{~K}$ temperature regime and present proof-of-principle measurements of the first prototype devices. We suggest that they are potentially more practical for multiplexing than the hotspot bolometers, for which two-stage thermal circuits are proposed to

*Address correspondence to this author at the VTT Technical Research Centre of Finland, P.O. Box 1000, FI-02044 VTT, Espoo, Finland;

Tel: +358-40-195 1482; E-mail: andrey.timofeev@vtt.fi overcome the problem of limited power gain bandwidth $[8$, 9].

\section{SUSPENDED SIS JUNCTION AS A BOLOMETER}

Our devices utilize Nb-AlOx-Nb SIS junction as a thermometer heated by the radiation via a separate suspended resistive element thermally coupled to the suspended junction. Such an arrangement allows for independent optimization of optical coupling matching to the absorber and electrical impedance matching to the read-out amplifier unlike in conventional SIS detectors or hotspot bolometers presently used in $4 \mathrm{~K} \mathrm{THz}$ scanners. The temperature sensitivity $d I / d T$ of an ideal SIS junction can be numerically derived by calculating net quasipartcile current $I$ across the tunnel barrier of resistance $R_{T}$ with standard single-electron tunneling approach assuming BCS type of density of states (DOS) in a superconductor. A finite DOS within the superconducting gap $[10,11]$ is commonly used to account for an excess current - a well-known performance-limiting factor for real tunnel junctions. This excess current persists through the sub-gap energy states which originate due to many reasons [12] such as microscopic imperfections both in a tunnel barrier and in a superconductor, high-order tunnelling processes, dissipative high-frequency electromagnetic environment [12], quasiparticle lifetime broadening due to the imaginary part of superconducting energy gap [11, 13], to mention few of those. In our case reasonable junction quality is required to have the excess current lower than the thermally excited quasiparticle current in order to minimize the contribution of the former one to the shot noise $S_{I}=2 e I \operatorname{coth}\left(e V / 2 k_{\mathrm{B}} T\right)$.

The electrical noise equivalent power NEP with noise contributions from the detector [14] and the readout circuit $[15]$ is then written as 
$\mathrm{NEP}^{2}=\mathcal{R}^{-2}\left[S_{I}+\frac{2 k_{B} T_{n, a}}{R_{d}}\left(\frac{R_{d}}{R_{a}}+\frac{R_{\mathrm{a}}}{R_{d}}\right)\right]+4 k_{B} T^{2} G$,

where $\mathcal{R}=G^{-1} d I / d T$ is the current responsivity of the detector, $G$ is the thermal conductance from the junction to the substrate and $R_{d} \equiv d V / d I$ is the dynamic resistance of the junction. The noise temperature and the optimum noise matching impedance of the readout amplifier are $T_{n, a}=\left(S_{V, a} S_{I, a}\right)^{1 / 2} / 2 k_{B}$ and $R_{a}=\left(S_{V, a} / S_{I, a}\right)^{1 / 2}$, respectively. Here $S_{V, a}^{1 / 2}$ and $S_{I, a}^{1 / 2}$ are the input referred equivalent voltage and current noise spectral densities of the amplifier. The first (second) term in the brackets of (1) is the shot noise (amplifier noise) contribution. With small $G$ the bolometer becomes phonon noise limited (the last term of (1)) while the phonon noise contribution itself also decreases as discussed in detail below (Fig. 4). Low resistance junctions are preferable in order to minimize the shot-noise contribution $\sqrt{S_{I}} / \mathcal{R} \propto \sqrt{R_{T}}$. Another noticeable fact is that a typical dynamic resistance of a few $\mathrm{kOhm}$ for $\mathrm{Nb}-\mathrm{AlOx}-\mathrm{Nb}$ junctions at $4 \mathrm{~K}$ allows the noise matching $\left(R_{d} \approx R_{a}\right)$ of the detector and the preamplifier.

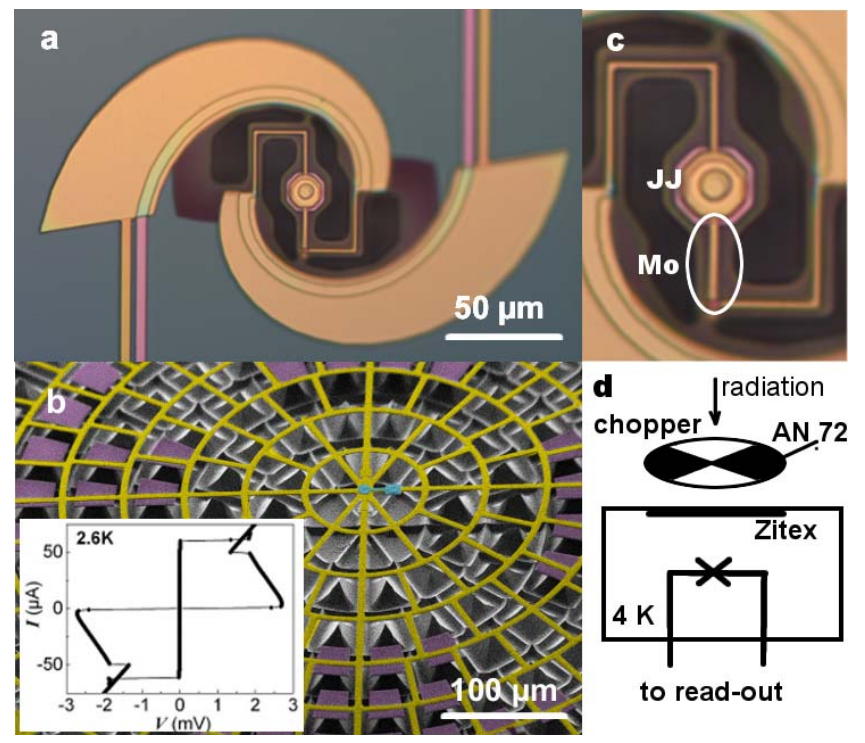

Fig. (1). (a) Logarithmic spiral antenna with suspended tunnel junction bolometer and Mo resistor. (b) Spiderweb $\mathrm{Nb} / \mathrm{Mo}$ bolometer with a tunnel junction in the center with a $I V$-curve at 2.6 $\mathrm{K}$ in the inset. (c) Zoomed image of the $\mathrm{JJ}$ and the resistor. (d) Measurement schematics of optical response of the bolometer.

\section{FABRICATION AND EXPERIMENTAL SETUP}

The bolometer structures have been fabricated based on $\mathrm{Nb}-\mathrm{AlOx}-\mathrm{Nb}$ trilayer junction SQUID process with junctions size of $6 \mu \mathrm{m}$ in diameter [16]. An additional fabrication step of isotropic $\mathrm{SF}_{6}$ plasma etch was applied to release the structures from the substrate. Molybdenum was used as the resistive material in the radiation absorbing layers. While these devices have been intended for electrical characterization only, we have investigated structures with two types of absorbing elements: a logarithmic-spiral antenna with a suspended stack of a SIS junction coupled to a heater (Fig. 1a, c) in the spirit of bridge bolometers [17], and a suspended spider-web absorber with a junction in the middle (Fig. 1b). All the suspended parts are supported from below by a silicon oxide layer. The working junctions for the spider web structures were found in a separate cooldown test, for which the $I V$ data at $2.6 \mathrm{~K}$ is presented in the inset of Fig. (1b). Below we will present more detailed measurement results on the logarithmic antenna structure.

The log-spiral antenna bolometer was tested in a cryogen-free pulse tube refrigerator. For signal read-out we have used low noise differential-pair FET-based amplifier electronics [18] $\left(S_{V, a}^{1 / 2} \approx 0.8 \mathrm{nV} / \mathrm{Hz}^{1 / 2}\right.$ and $S_{I, a}^{1 / 2} \lesssim 15$ $\mathrm{fA} / \mathrm{Hz}^{1 / 2}$, with these values we find $R_{a} \geq 53 \mathrm{k} \Omega$ and $T_{n, a} \lesssim$ $0.4 \mathrm{~K})$. We applied a readout method including voltage bias and current readout based on active feedback (schematically shown in Fig. 2a) allowing for stable voltage biasing of the junction within the gap without magnetic suppression of the Josephson coupling.

\section{MEASUREMENTS}

The mixed-down noise component due to Josephson oscillations is estimated to be orders of magnitude smaller than other noise contributions in the operating range of biasing voltages. The tunnel resistance of the junction is measured to be $R_{T}=120 \Omega$ with the sub-gap quasiparticle current $I=1 \mu \mathrm{A}$ at bias voltage $V=1.5 \mathrm{mV}$ at $4.4 \mathrm{~K}$ (Fig. 2a). From numerically calculated $I V$ curves using ideal BCS-gap model we find quasiparticle current $I=0.59 \mu \mathrm{A}$ $\left(e I R_{T}=0.05 \Delta(0)\right.$ at $4.4 \mathrm{~K}$ with $\left.\Delta(0)=1.4 \mathrm{meV}\right)$. This indicates that the excess leakage current in the junction is about $0.4 \mu \mathrm{A}$ in contrast to near-BCS limited behavior of non-released junctions fabricated with the same junction process [19]. This may be due to the release-etching step, though its only effect is to enhance the shot noise by about a factor of $\sqrt{2}$ as compared to that of the ideal junction.

From the measured IVs (Fig. 2a) at various bath temperatures we obtain responsivity $d I / d T=0.57 \mu \mathrm{A} / \mathrm{K}$ at $V=1.4 \mathrm{meV}$ at $4.4 \mathrm{~K}$, in accordance with its value 0.58 $\mu \mathrm{A} / \mathrm{K}$ derived from temperature dependent IVs computed with ideal BCS model. Fig. (2a) also shows that, in the subgap region, the responsivity depends only weakly on the temperature and bias voltage. The maximum of the dynamic resistance of $7 \mathrm{k} \Omega$ (Fig. 2b) is achieved at the optimal bias point $V_{\text {opt }}=1.7 \mathrm{mV}$, i.e. only about a factor of 4 times smaller than the noise matched case. The measured total current noise $I_{N}$ referred to the amplifier input (averaged over the readout band $4.5-5.0 \mathrm{kHz}$ ) is plotted against biasing voltage in Fig. (2c) at a few bath temperatures. From this figure the contribution of the thermal noise of the feedback resistor $R_{\mathrm{fb}}=30 \mathrm{k} \Omega$ corresponding to current noise of about $0.7 \mathrm{pA} / \mathrm{Hz}^{1 / 2}$ has been subtracted. The feedback noise is a practical flaw in our test system avoidable by proper selection of $R_{\mathrm{fb}}$, or by cooling down the resistor. The lowest input current noise $I_{\mathrm{N}}=0.6 \mathrm{pA} / \mathrm{Hz}^{1 / 2}$ is 
achieved at $V_{\text {opt }}$ at $4.4 \mathrm{~K}$. Also, in a wide range of voltage biases 1.2-2.2 $\mathrm{mV}$ around $V_{\mathrm{opt}}$ the noise is detectorlimited and the amplifier noise contribution is negligible. Only in low- $R_{d}$ operating points the amplifier matching becomes worse and the amplifier voltage noise becomes dominant.
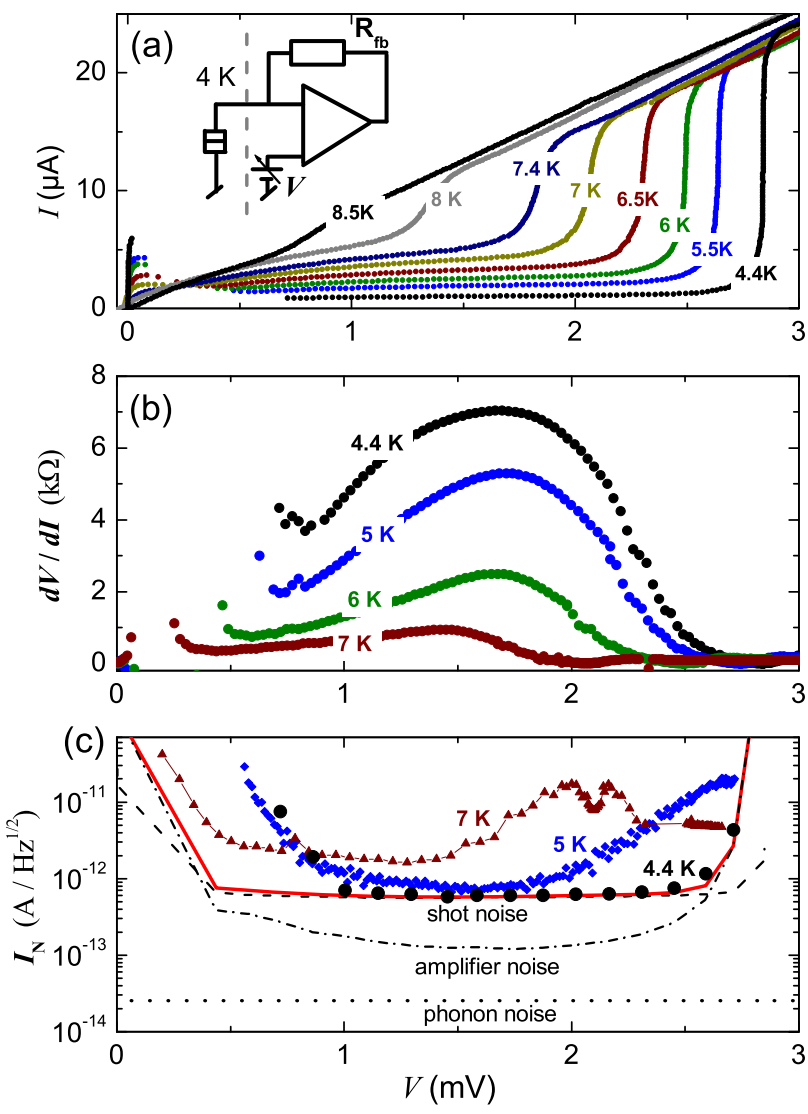

Fig. (2). (a) Measured $I V$-curves at 4.4...8.6 K. (b) Dynamic resistance derived from the measured $I V$ 's at a few bath temperatures. (c) Averaged input current noise spectral density measured against bias voltage at a few bath temperatures (dots). The lines show current noise contributions calculated for $T=4.4 \mathrm{~K}$ with Eq. (1) for $(I, V)$ and $R_{d}$ values taken from measured $I V$ data, with $d I / d T=0.57 \mu \mathrm{A} / \mathrm{K}, R_{a}=53 \mathrm{k} \Omega, T_{n, a}=0.4 \mathrm{~K}$, $G=400 \mathrm{nW} / \mathrm{K}$ as in the experiment. Red line shows the total input current noise. Dashed lines show the contributions of shot noise (dashed), amplifier noise (dashed-dotted) and the phonon noise (dotted).

To determine $G$, we have performed thermal conductance measurements on the test structures of suspended junctions with the heater resistors. The heater was driven by a small DC power and the total thermal conductance was inferred by comparing the junction responses to elevated temperature and induced heater power. Based on the geometry we estimated the thermal conductance between the junction and the log-antenna to be $G \simeq 400 \mathrm{nW} / \mathrm{K}$. Assuming $0.1 \mathrm{~W} /(\mathrm{m} \cdot \mathrm{K})$ thermal conductivity for $\mathrm{SiO}_{2}$ from the literature [20], about 140 $\mathrm{nW} / \mathrm{K}$ of this comes from the $\mathrm{SiO}_{2}$ layer including unwanted parasitic thermal links (see Fig. 1c) that remained after the etch-release fabrication step due to the finite $2 \mu \mathrm{m}$ contactlithography resolution. The rest of $260 \mathrm{nW} / \mathrm{K}$ originates from an unoptimized thickness of $650 \mathrm{~nm}$ of $\mathrm{Nb}$ leads. From these data we estimated $4 \mathrm{~K}$ thermal conductivity $6.7 \mathrm{~W} /(\mathrm{m} \cdot \mathrm{K})$ of $\mathrm{Nb}$. This value is close to the Wiedemann-Franz thermal conductivity $6.5 \mathrm{~W} /(\mathrm{m} \cdot \mathrm{K})$ with the resistivity $3.6 \times 10^{-8}$ $\Omega \mathrm{m}$ at $10 \mathrm{~K}$, reduced by a factor of 5 with respect to the measured $300 \mathrm{~K}$ resistivity. With $G=400 \mathrm{nW} / \mathrm{K}$ the electrical $\mathrm{NEP}=I_{N} / \mathcal{R}$ reads $\simeq 4 \times 10^{-13} \mathrm{~W} / \mathrm{Hz}^{1 / 2}$ (Fig. 4a) and the detector operates in the shot noise limit.

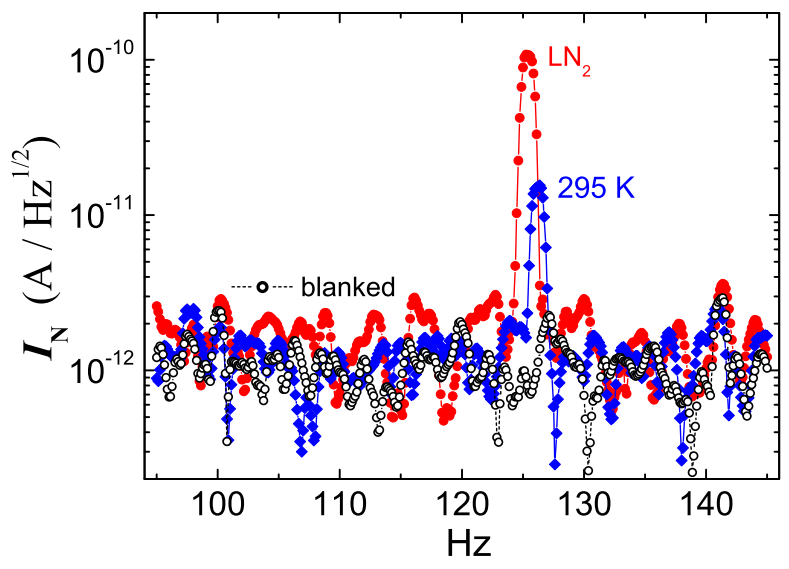

Fig. (3). Optical response of the log-antenna bolometer seen as a spike at the chopping frequency of $125 \mathrm{~Hz}$ in the $50 \mathrm{~Hz}$ band of the noise spectrum.

We have also carried out preliminary optical response measurements against room temperature and liquid nitrogen temperature backgrounds using a chopper at the frequency of $125 \mathrm{~Hz}$ (Fig. 1d). Zitex [21] filters in cryostat windows provided a low pass cutoff at $\sim 1 \mathrm{THz}$. We observed a pronounced optical signal in the noise spectrum at the corresponding chopping frequency as shown in Fig. (3). The signal from the liquid nitrogen background was about one order of magnitude stronger than the room temperature background. Since our prototype bolometers chips were designed mainly for electrical characterization, we give only the rough estimate for the radiometric noise equivalent temperature difference (NETD $\sim \Delta T / \mathrm{SNR}$ ) from the SNR ratios found from the power levels of the optical signals in Fig. (3). SNR $\sim 130$ for liquid nitrogen backgrounds with $\Delta T \sim 300-77 \sim 200 \mathrm{~K})$. Thus NETD $\simeq 1-2 \mathrm{~K}$ for $1 \mathrm{~s}$ integration time. This is the expected radiometric temperature resolution [22] as for lithographic antenna bolometer structures used with no lens and front-side radiation facing the optical coupling is very poor $\left(\leq 10^{-3}\right)$. With improved optical coupling the SNR ratios are expected to be of at least 100 times higher to become performance competitive with STJ detectors at $4.2 \mathrm{~K}$ reported in ref. [4]. 

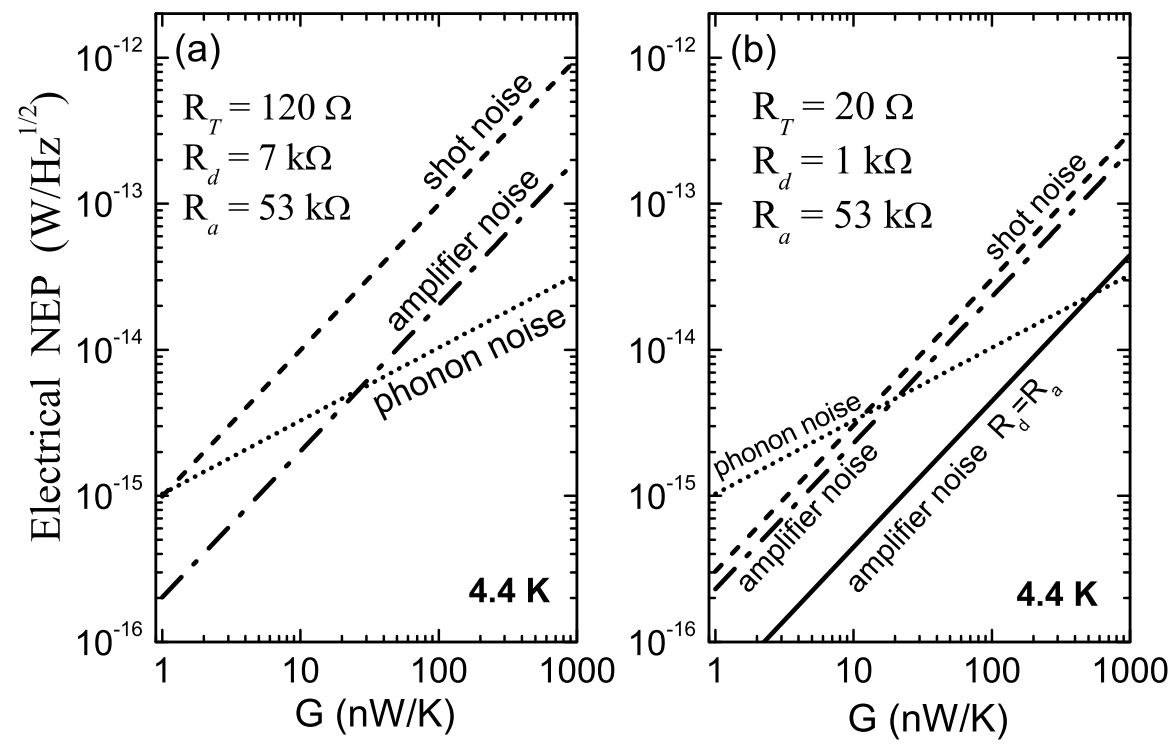

Fig. (4). (a) Calculated (with Eq. (1), $I=1 \mu \mathrm{A}, d I / d T=0.57 \mu \mathrm{A} / \mathrm{K}$ ) shot noise (dashed), amplifier noise (dashed-dotted) and phonon noise (dotted) contributions to electrical NEP $v s G$ at $4.4 \mathrm{~K}$ with the amplifier and junction parameters as in the experiment: $T_{n, a}=0.4 \mathrm{~K}$, $R_{a}=53 \mathrm{k} \Omega ; R_{d}=7 \mathrm{k} \Omega, R_{T}=120 \Omega$. (b) Optimized case for $R_{T}=20 \Omega, T_{n, a}=0.4 \mathrm{~K}$ and $R_{d}=(1 / 53) R_{a}$. The solid line shows the noise matched limit $R_{d}=R_{a}$.

\section{MULTIPLEXING RESOLUTION}

\subsection{Resolution of an Optimized Single Pixel}

With an optimized detector design, a thermal conductance of $10 \mathrm{nW} / \mathrm{K}$ at $4 \mathrm{~K}$ can be realized by replacing the thick $\mathrm{Nb}$ wire with thin $\mathrm{NbN}$. Near $T_{c}, \mathrm{NbN}$ has a thermal conductivity of 1 -- 2 orders of magnitude lower than $\mathrm{Nb}$ [23]. Increasing transition temperature (or reducing the operation temperature) further decreases the electronic contribution to $G$, which scales as $\propto \exp \left(-T / T_{c}\right)$ at $T \ll T_{c} . G=10 \mathrm{nW} / \mathrm{K}$ would provide operating close to the phonon noise limit with $\mathrm{NEP} \simeq 10^{-14} \mathrm{~W} / \mathrm{Hz}^{1 / 2}$ at $4 \mathrm{~K}$ and further reduction of the amplifier contribution (Fig. 4a) allowing multiplexing of larger arrays. This figure is comparable to the electrical NEP of the detectors used in a functional video-rate $\mathrm{THz}$ scanner [1]. We also note that in order to operate entirely in the phonon noise limit with NEP $\leq 3 \times 10^{-15} \mathrm{~W} / \mathrm{Hz}^{1 / 2}$, junctions with lower $R_{T}$ (higher $d I / d T$ ) have to be used. However, this will also reduce $R_{d}$ resulting in a larger mismatch. As a compromise we give the calculated NEP in Fig. (4b) for a detector with $R_{T}=20 \Omega$ and $R_{d}=1 \mathrm{k} \Omega$ assuming the present preamplifier $R_{a}=53$ $\mathrm{k} \Omega$. In addition, a noise line for an impedance matched preamplifier with the same noise temperature of $0.4 \mathrm{~K}$ is given. Choosing the room temperature preamplifier with lower $R_{a}$ becomes a trade-off between keeping both sufficient noise matching and low enough $T_{n, a}$. Another alternative is to use cryogenic amplifiers [24]. Nevertheless, with multiplexing the NEP requirement is less restrictive since all the pixels are averaged continuously, thus in terms of electrical NEP our experimental results (Fig. 4a) can be sufficient.

\subsection{Multiplexing Scheme}

Next, we analyze the feasibility of time domain multiplexing based on a cryogenic MUX switch and on a semiconducting amplifier with the feedback circuit as in our experiment (Fig. 5). A cold CMOS MUX-switch can be fabricated with adequate noise parameters and heat dissipation as low as $\simeq 1 \mu \mathrm{W} /$ channel [25].

Assuming frame period $T_{0}$ and readout bolometer time $\Delta t$, the maximum number of multiplexed detectors is $N=T_{0} / \Delta t$. A capacitor $C$ is added in parallel to the detector to integrate the signal electrically and to maintain the operating point when the amplifier is not connected. The value of $C$ is chosen in such a way that during the off-time of the readout the detector voltage drop $\Delta V \sim I T_{0} / C$ is much smaller than the operating voltage $V$.

The requirement on $C$ can be relieved by introducing an additional bias circuit for compensating the leakage current as shown in Fig. (5). In this scheme the detector is voltage biased during the time of the corresponding switch being closed but current biased otherwise. Even in the case of full dark-current compensation we still need to integrate the detector noise. This is required if the thermal time constant of the detectors cannot be increased due to practical reasons or if shot noise is comparable to phonon noise and thus needs to be integrated. With dark-current compensation the capacitance value needed becomes

$C \geq T_{0} / R_{d}$ 


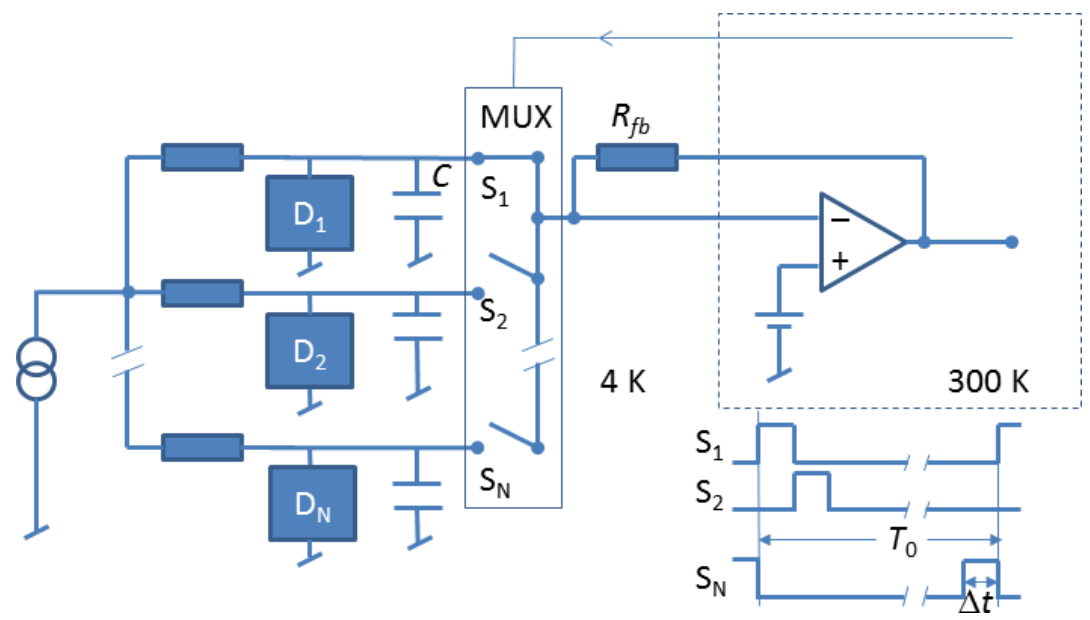

Fig. (5). A Possible multiplexing scheme. The bolometers are voltage-biased during read-out time $\Delta t$ and current-biased in the off-state to compensate for the leakage current. The bias resistors larger than $R_{d}$ provide uniform bias of the bolometers.

This allows to use lower $C$ which is favorable for a circuit design.

\subsection{Multiplexing Penalty Due to Readout Noise}

The capacitance $C$ provides the additional complication that it makes the impedance seen by the readout amplifier frequency dependent. In the following we derive an expression that takes into account the effect of such frequency dependent source impedance on the power resolution.

We describe the signal as the current integrated over the scanning period, i.e. as charge

$Q_{d}=(d I / d T) P T_{0} / G$,

where $P$ is the detected signal power. We calculate the corresponding charge noise $\sigma_{A, Q}$ due to the voltage noise of the preamplifier in terms of Allan variance (see e.g. [26]).

The calculation is given in the appendix. Equating $Q_{d}$ with the Allan deviation, we get the power resolution due to a voltage noise limited amplifier

$\sigma_{A, P}=\frac{G \sigma_{A, Q}}{(d I / d T) T_{0}}=\frac{G C \sqrt{2 S_{V, a} f_{\max }}}{T_{0}(d I / d T)}$.

Here $f_{\max }$ is the cutoff frequency of the electronics. Although the noise spectrum is not white, we give an effective power spectral density figure with respect to the full frame time $T_{0}$ to help to compare with the detector noise of Eq. ((1))

$S_{P}=2 T_{0} \sigma_{A, P}^{2}=\left(\frac{2 G C}{d I / d T}\right)^{2} \frac{S_{V, a} f_{\max }}{T_{0}}$.

Current noise can usually be neglected in the case of CMOS or JFET amplifiers and in any case its treatment is trivial.

Defining parameters $\quad \alpha=R_{d} C / T_{0} \geq 1 \quad$ and $\beta=f_{\max } \Delta t=f_{\max } T_{0} / N>1$, we get
$\sigma_{A, P}=\sqrt{\frac{2 S_{V, a} \beta N}{T_{0}}} \frac{\alpha G}{R_{d} d I / d T}$.

Capacitive biasing adds thus a factor $\alpha \sqrt{\beta}>1$ to the standard multiplexing penalty $\sqrt{N}$ of time-division multiplexing.

As an example, let $N=100$ pixels and $T_{0}=30 \mathrm{~ms}$ for video rate. With an improved detector design and high quality junctions it is possible to get $R_{d} \approx 10 \mathrm{k} \Omega$, $d I / d T=3.5 \mu \mathrm{A} / \mathrm{K}, G=10 \mathrm{nW} / \mathrm{K}, \sqrt{S_{V, a}}=0.8 \mathrm{nV} / \sqrt{\mathrm{Hz}}$, $\alpha=1$ and $\beta=3$. Eq. ((5)) then gives

$\mathrm{NEP} \simeq 30 \mathrm{fW} @ 30 \mathrm{~ms}$

with corresponding effective PSD

$\sqrt{S_{P}} \simeq 8 \mathrm{fW} / \sqrt{\mathrm{Hz}}$.

These figures are well sufficient for wideband passive THz imaging [1].

\section{SUMMARY}

We have demonstrated suspended SIS tunnel junction bolometers operating in a $4 \mathrm{~K}$ temperature regime. Our first prototype devices perform in a shot noise limit. With introducing thinner superconducting materials together with low resistance junctions and proper optical coupling the NETD of the bolometers performance is expected to be in the ballpark of $\sim 1-10 \mathrm{mK}$. Furthermore, the fact that the noise in the SIS-bolometer device is strongly dominated by the detector itself rather than by the read-out setup, suggests that they are suitable for multiplexing. The possibilities of a wide-band $\mathrm{THz}$ signal matching and good amplifier matching make SIS-bolometer a promising candidate for large array imagers.

\section{ACKNOWLEDGEMENT}

We thank J. Penttilä, M. Grönholm, H. Sipola, A. Rautiainen, M. Kiviranta and M. Leivo for useful 
discussions and help with the experimental setup. This work is financially supported by the Academy of Finland Grant No. 140484 (Cryogenic Microsensors), and Finnish Academy Program for Center of Excellence in Low Temperatures and Quantum Devices.

\section{CONFLICT OF INTEREST}

The authors confirm that this article content has no conflicts of interest.

\section{APPENDIX}

\section{Charge Fluctuations Due to Voltage Noise on a Capacitive Load}

The source impedance seen by the preamplifier is frequency dependent, which complicates the noise analysis. We give here a calculation based on charge balance and Allan variance between successive measurements. With Allan variance temporal variations of a stochastic process can be linked to its power spectral density. This analysis is applicable to other time domain multiplexing cases with frequency dependent source impedance. In our case the source impedance is dominated by the capacitance $C$

$\left|Z_{\omega}\right|=1 / \omega C$.

During time $T_{0}$, the voltage over the detector changes by $\Delta V$

$Q_{d}=C \Delta V \simeq I T_{0}$,

where $I$ is average signal current and uncompensated leakage current through the bolometer. This is compensated by a loading current $I_{a}$

$Q_{l}=Q_{d}=I_{a} \Delta t$

during the charging/readout period $\Delta t$.

The charge noise driven into the capacitor during loading time is the noise current $i(t)$ caused by the voltage noise of the readout, integrated over time $\Delta t$

$q_{l}=\int_{0}^{\Delta t} i(t) d t$.

Let us compare this to a neighboring measurement and calculate the Allan variance [26]. We get

$$
\begin{aligned}
\sigma_{A, Q}^{2} & =\left\langle\left(q_{j+1}-q_{j}\right)^{2}\right\rangle / 2=\left\langle q_{j}^{2}-q_{j} q_{j+1}\right\rangle \\
& =\iint i(t) i\left(t^{\prime}\right) d t d t^{\prime}-\iint i(t) i\left(T_{0}+t^{\prime}\right) d t d t^{\prime} \\
& =\int_{0}^{\infty} \frac{d f}{(\pi f)^{2}} S_{I_{V}}(f) \sin ^{2}(\pi f \Delta t)\left(1-\cos \left(2 \pi f T_{0}\right)\right),
\end{aligned}
$$

where time integration intervals are from 0 to $\Delta t$. Here

$$
S_{I_{V}}=S_{V, a} /\left|Z_{s}\right|^{2}=(2 \pi)^{2} S_{V, a} C^{2} f^{2}
$$

is the power spectral density of the current noise caused by the voltage noise $S_{V, a}$. The result diverges for a noise source with infinite bandwidth. In practice a high frequency cutoff $f_{\max }$ always exists. To avoid signal crosstalk between detectors, $f_{\max }>1 / \Delta t$. Assuming $\Delta t<T_{0}$, we end up at

$$
\sigma_{A, Q}^{2} \simeq 2 S_{V, a} C^{2} f_{\max } \text {. }
$$

\section{REFERENCES}

[1] Luukanen A, Grönberg L, Grönholm M, et al. Real-time passive terahertz imaging system for standoff concealed weapons imaging. 5-9 April, 2010. Proceedings of SPIE Defense, Security, and Sensing Conference. Orlando, Florida, USA 2010; 7670 : 767004.

[2] Grossman E, Dietlein C, Ala-Laurinaho J, et al. Passive terahertz camera for standoff security screening. Appl Opt 2010; 49: E10620.

[3] E. Heinz E, Born D, Zieger G, et al. Progress report on Safe VISITOR: approaching a practical instrument for terahertz security screening. Proceedings of SPIE Defense, Security, and Sensing Conference 2010; 7670: 767005.

[4] Ariyoshi S, Otani C, Dobroiu A, et al. Terahertz imaging with a direct detector based on superconducting tunnel junctions. Appl Phys Lett 2006; 88: 203503.

[5] Prober D, Teufel J, Frunzio L, Wilson C, Schoelkopf R. Quasiparticle dynamics and a new, high-resolution readout of STJ photon detector. Nucl Inst Methods Phys Res A 2006; 559: 676-9.

[6] Ariyoshi S, Taino T, Dobroiu A, et al. Terahertz detector based on a superconducting tunnel junction coupled to a thin superconductor film. Appl Phys Lett 2009; 95: 193504.

[7] Naruse M, Sekimoto Y, Suzuki T. et al. Design of SIS imaging array for terahertz astronomy. 34th International Conference. IRMMW-THz. 2009; pp.1-2.

[8] Helistö P, Hassel J, Luukanen A, Seppä H. Superconducting transition detectors as power amplifiers for cryomultiplexing. 19th International Symposium. ISSTT 2008; Abstract P5-2.

[9] Helistö P, Hassel J, Luukanen A, Seppä H. Superconducting transition detector in power amplification mode. Supercond Sci Technol 2009; 22: 045004.

[10] Dynes R, Narayanamurti V, Garno J. Direct Measurement of Quasiparticle-Lifetime Broadening in a Strong-Coupled Superconductor. Phys Rev Lett 1978; 41: 1509.

[11] Mitrovic B, Rozema L. On the correct formula for the lifetime broadened superconducting density of states. J Phys Condens Matter 2008; 20: 015215.

[12] Pekola J, Maisi V, Kafanov S, et al. Environment-assisted tunneling as an origin of the dynes density of states. Phys Rev Lett 2011; 105: 026803. See also references therein.

[13] Noguchi T, Suzuki T, Endo A, et al. Effect of lifetime broadening of superconducting energy gap on quasiparticle tunneling current. 20th ISSTT 2009; pp. 74-9.

[14] Giazzoto F, Heikkilä T, Luukanen A, Savin A, Pekola J. Opportunities for mesoscopics in thermometry and refrigeration: Physics and applications. Rev Mod Phys 2006; 78: 2006.

[15] Ryhänen T, Seppä H. SQUID magnetometers for low-frequency applications. J Low Temp Phys 1989; 76: 5-6.

[16] Grönberg L, Hassel J, Helistö P, Ylilamm M. Fabrication process for RSFQ/Qubit Systems. IEEE Trans Appl Supercond 2007; 17(2): 952.

[17] Luukanen A, Pekola J. A superconducting antenna-coupled hotspot microbolometer. Appl Phys Lett 2003; 82: 223970.

[18] Penttilä J, Sipola H, Helistö P, Seppä H. Low-noise readout of superconducting bolometers based on electrothermal feedback. Supercond Sci Technol 2006: 19(4): 319.

[19] Castellano G, Grönberg L, Carelli P, et al. Characterization of a fabrication process for the integration of superconducting qubits and rapid-single-flux-quantum circuits. Supercond Sci Technol 2006; 19: 860-4.

[20] Ekin JW. Experimental techniques for low temperature measurements. USA: Oxford University Press 2006.

[21] Benford DJ, Gaidis MC, Kooi JW. Optical properties of Zitex in the infrared to submillimeter. Appl Opt 2003; 42(25): 5118-22.

[22] Rebeiz GM. Millimeter-wave and terahertz integrated circuit antennas. Proc IEEE 1992; 80(11): 1748-70.

[23] Dietlein C, Luukanen A, Penttilä JS, et al. Performance comparison of $\mathrm{Nb}$ and $\mathrm{NbN}$ antenna-coupled microbolometers. SPIE Proc 2007; 6549: 65490M. 
[24] Kiviranta M. Use of SiGe bipolar transistors for cryogenic readout of SQUIDs. Supercond Sci Technol 2006; 19: 1297-302.

[25] (a) Hibi Y, Matsuo H, Arai H, Nagata H, Ikeda H, Fujiwara M. The cryogenic multiplexer and shift register for submillimeter-wave digital camera. Cryogenics 2009; 49(11): 672-5. (b) Matsuo H, Hibi Y, Nagata H, Ikeda H, Fujiwara M. SISCAM 32-ch cryogenic readout module with GaAs-JFET ASICs. Proc SPIE 2010; 7741: $77412 \mathrm{~V}$.

[26] Helistö P, Seppä H. Minimum variance method for treatment of measurements with low-frequency noise. Metrologia 2001; 38: 489-96.

(C) Timofeev et al.; Licensee Bentham Open.

This is an open access article licensed under the terms of the Creative Commons Attribution Non-Commercial License (http://creativecommons.org/licenses/by$\mathrm{nc} / 3.0 /$ ) which permits unrestricted, non-commercial use, distribution and reproduction in any medium, provided the work is properly cited. 